\title{
Sharpe-ratio pricing and hedging of contingent claims in incomplete markets by convex programming ${ }^{\Uparrow}$
}

\author{
Mustafa Ç. Pınar* \\ Department of Operations Research and Financial Engineering, Princeton University, Princeton, NJ 08544, United States
}

Received 7 January 2007; received in revised form 25 November 2007; accepted 29 November 2007

Available online 21 February 2008

\begin{abstract}
We analyze the problem of pricing and hedging contingent claims in a financial market described by a multi-period, discrete-time, finite-state scenario tree using an arbitrage-adjusted Sharpe-ratio criterion. We show that the writer's and buyer's pricing problems are formulated as conic convex optimization problems which allow to pass to dual problems over martingale measures and yield tighter pricing intervals compared to the interval induced by the usual no-arbitrage price bounds. An extension allowing proportional transaction costs is also given. Numerical experiments using $S \& P 500$ options are given to demonstrate the practical applicability of the pricing scheme.
\end{abstract}

(C) 2008 Elsevier Ltd. All rights reserved.

Keywords: Contingent claim; Pricing; Hedging; Sharpe ratio; Martingales; Transaction costs; Convex programming

\section{Introduction}

A fundamental question of financial economics is to price an uncertain future stream of payments, i.e. a stochastic future cash flow. The prevailing approach to pricing is to replicate the uncertain cash flow using existing financial instruments and to find a price relative to these instruments so as to avoid an arbitrage opportunity in the financial market (hedging). When the financial market is complete, i.e. all stochastic future cash flows can be replicated using existing liquid instruments this approach yields unique sets of prices without any assumptions about individual investor's preferences. Ross (1976, 1978) proves that the no-arbitrage condition is equivalent to the existence of a linear pricing rule and positive state prices that correctly value all assets. This linear pricing rule comes from the risk neutral probability measure in the Cox-Ross option pricing model; Harrison and Kreps (1979) showed that the linear pricing operator is an expectation taken with respect to a martingale measure.

\footnotetext{
This paper was not presented at any IFAC meeting. This paper was recommended for publication in revised form by Editor Berç Rustem.

* Corresponding address: Department of Industrial Engineering, Bilkent University, 06800 Ankara, Turkey.

E-mail addresses: mpinar@princeton.edu, mustafap@bilkent.edu.tr.
}

The pricing problem is complicated by the fact that most financial markets are incomplete, i.e. not all future uncertain cash flows can be replicated exactly using the existing instruments. This observation leads to a wealth of literature on pricing and hedging in incomplete markets; see e.g., Bernardo and Ledoit (2000), Carr, Geman, and Madan (2001), Černý and Hodges (2000) and Staum (2004). A common approach is to find the cheapest portfolio dominating a stochastic future cash flow and the most expensive portfolio dominated by it, and use these respective values as bounds on the price of the stochastic cash flow. These bounds are referred to as super-replication and sub-replication bounds or no-arbitrage (or equilibrium) bounds . We use these bounds as a benchmark in the present paper.

Against this background, the purpose of this paper is to investigate how an arbitrage-adjusted Sharpe-ratio criterion (Černý, 2004; Judice, 2005) and convex optimization can help us develop a simple valuation framework in an incomplete market. We show in a multi-period framework that in the absence of arbitrage (i.e. in the absence of infinite Sharpe ratios) while aiming for a finite Sharpe ratio and giving up a totally risk averse attitude in the non-negative terminal wealth positions, price bounds tighter than the noarbitrage bounds to the contingent claim can be computed in an incomplete market. The present work is inspired by the contributions of Cochrane and Saa-Requejo (2000) and those of 
Černý $(2003,2004)$ where the authors develop similar ideas but essentially departing from the dual space of pricing measures. We start from the more natural primal hedging space which is the domain where investors operate rather than from the space of martingale measures. In the simple framework of discrete-time, multiperiod, finite state probability framework resulting in a scenario tree, after laying the background in Section 2 we show in Section 3 how the problems of writers and buyers are formulated as convex (conic) optimization problems, and pass to the results of Černý (2003) and Cochrane and Saa-Requejo (2000) using convex programming duality. The conic programming approach of the present paper has the advantage of accommodating imperfections including taxes, transaction costs, and other regulatory or institution specific requirements in a more flexible manner than other approaches. We extend the approach to include proportional transaction costs in Section 4. To demonstrate the computational feasibility of the pricing approach advocated in this paper in a practical setting, in Section 5 we report the results of realistic numerical experiments on $S \& P 500$ index options data and using offthe-shelf interior point software, and compare them with those of King, Koivu, and Pennanen (2005) based on no-arbitrage pricing. We conclude with a discussion and future research problems in Section 6.

\section{The stochastic scenario tree, arbitrage, martingales and pricing bounds}

As in King (2002) we assume that security prices and other payments are discrete random variables supported on a finite probability space $(\Omega, \mathcal{F}, P)$ whose atoms are sequences of realvalued vectors (asset values) over the discrete time periods $t=0,1, \ldots, T$. We further assume the market evolves as a discrete, non-recombinant scenario tree (hence, suitable for incomplete markets) in which the partition of probability atoms $\omega \in \Omega$ generated by matching path histories up to time $t$ corresponds one-to-one with nodes $n \in \mathcal{N}_{t}$ at level $t$ in the tree. The set $\mathcal{N}_{0}$ consists of the root node $n=0$, and the leaf nodes $n \in \mathcal{N}_{T}$ correspond one-to-one with the probability atoms $\omega \in \Omega$. In the scenario tree, every node $n \in \mathcal{N}_{t}$ for $t=1, \ldots, T$ has a unique parent denoted $\pi(n) \in \mathcal{N}_{t-1}$, and every node $n \in \mathcal{N}_{t}, t=0,1, \ldots, T-1$ has a non-empty set of child nodes $\mathcal{S}(n) \subset \mathcal{N}_{t+1}$. We denote the set of all nodes in the tree by $\mathcal{N}$. The probability distribution $P$ is obtained by attaching positive weights $p_{n}$ to each leaf node $n \in \mathcal{N}_{T}$ so that $\sum_{n \in \mathcal{N}_{T}} p_{n}=1$. For each non-terminal (intermediate level) node in the tree we have, recursively, $p_{n}=\sum_{m \in \mathcal{S}(n)} p_{m}, \forall n \in \mathcal{N}_{t}, t=T-$ $1, \ldots, 0$. Hence, each intermediate node has a probability mass equal to the combined mass of the paths passing through it. The ratios $p_{m} / p_{n}, m \in \mathcal{S}(n)$ are the conditional probabilities that the child node $m$ is visited given that the parent node $n=\pi(m)$ has been visited.

A random variable $X$ is a real-valued function defined on $\Omega$. It can be lifted to the nodes of a partition $\mathcal{N}_{t}$ of $\Omega$ if each level set $\left\{X^{-1}(a): a \in \mathbb{R}\right\}$ is either the empty set or is a finite union of elements of the partition. In other words, $X$ can be lifted to $\mathcal{N}_{t}$ if it can be assigned a value on each node of $\mathcal{N}_{t}$ that is consistent with its definition on $\Omega$, King (2002). This kind of random variable is said to be measurable with respect to the information contained in the nodes of $\mathcal{N}_{t}$. A stochastic process $\left\{X_{t}\right\}$ is a time-indexed collection of random variables such that each $X_{t}$ is measurable with respect $\mathcal{N}_{t}$. The expected value of $X_{t}$ is uniquely defined by the sum $\mathbb{E}^{P}\left(X_{t}\right):=\sum_{n \in \mathcal{N}_{t}} p_{n} X_{n}$. The conditional expectation of $X_{t+1}$ on $\mathcal{N}_{t}$ is given by the expression $\mathbb{E}^{P}\left(X_{t+1} \mid \mathcal{N}_{t}\right):=\sum_{m \in \mathcal{S}(n)} \frac{p_{m}}{p_{n}} X_{m}$. Under the light of the above definitions, the market consists of $J+1$ tradable securities indexed by $j=0,1, \ldots, J$ with prices at node $n$ given by the vector $S_{n}=\left(S_{n}^{0}, S_{n}^{1}, \ldots, S_{n}^{J}\right)$. We assume as in King (2002) that the security indexed by 0 has strictly positive prices having the same value at every node of a given time step $t$. This asset corresponds to the risk-free asset in the classical valuation framework. Choosing this security as the numéraire, and using the discount factors $\beta_{n}=1 / S_{n}^{0}$ we define $Z_{n}^{j}=\beta_{n} S_{n}^{j}$ for $j=0,1, \ldots, J$ and $n \in \mathcal{N}$, the security prices discounted with respect to the numéraire. Note that $Z_{n}^{0}=1$ for all nodes $n \in \mathcal{N}$.

The amount of security $j$ held by the investor in state (node) $n \in \mathcal{N}_{t}$ is denoted $\theta_{n}^{j}$. Therefore, to each state $n \in \mathcal{N}_{t}$ is associated a vector $\theta_{n} \in \mathbb{R}^{J+1}$. We refer to the collection of vectors $\theta_{0}, \theta_{1}, \ldots, \theta_{|\mathcal{N}|}$ as $\Theta$. The value of the portfolio at state $n$ (discounted with respect to the numéraire) is $Z_{n} \cdot \theta_{n}=$ $\sum_{j=0}^{J} Z_{n}^{j} \theta_{n}^{j}$.

An arbitrage is a sequence of portfolio holdings that begins with a zero initial value (note that short sales are allowed), makes self-financing portfolio transactions throughout the planning horizon and achieves a non-negative terminal value in each state, while in at least one terminal state it achieves a positive value with non-zero probability. The self-financing transactions condition is expressed as $Z_{n} \cdot \theta_{n}=Z_{n} \cdot \theta_{\pi(n)}, n>0$.

Definition 1. If there exists a probability measure $Q=$ $\left\{q_{n}\right\}_{n \in \mathcal{N}_{\mathcal{T}}}$ such that

$Z_{t}=\mathbb{E}^{Q}\left(Z_{t+1} \mid \mathcal{N}_{t}\right) \quad(t \leq T-1)$

then the vector process $\left\{Z_{t}\right\}$ is called a vector-valued martingale under $Q$, and $Q$ is called a martingale probability measure for the process.

Definition 2. A discrete probability measure $Q=\left\{q_{n}\right\}_{n \in \mathcal{N}_{T}}$ is equivalent to a (discrete) probability measure $P=\left\{p_{n}\right\}_{n \in \mathcal{N}_{T}}$ if $q_{n}>0$ exactly when $p_{n}>0$.

King proved the following (c.f. Theorem 1 of King (2002)) result that is a re-statement of Theorem 1 of Harrison and Kreps (1979) in our setting (see also Ross $(1976,1978)$ ):

Theorem 1. The discrete state stochastic vector process $\left\{Z_{t}\right\}$ is an arbitrage free market price process if and only if there is at least one probability measure $Q$ equivalent to $P$ under which $\left\{Z_{t}\right\}$ is a martingale.

Consider now the pricing problem of a contingent claim $F$ with pay-offs $F_{n}, n>0$. The seller of the contingent claim is 
interested in solving the following problem (WA):

$$
\begin{array}{ll}
\min & Z_{0} \cdot \theta_{0} \\
\text { s.t. } & Z_{n} \cdot\left(\theta_{n}-\theta_{\pi(n)}\right)=-\beta_{n} F_{n}, \quad \forall n \in \mathcal{N}_{t}, t \geq 1 \\
& 0 \leq Z_{n} \cdot \theta_{n} \quad \forall n \in \mathcal{N}_{T},
\end{array}
$$

while the buyer's problem of finding the maximum acceptable price would be (BA)

$$
\begin{array}{ll}
\max & -Z_{0} \cdot \theta_{0} \\
\text { s.t. } & Z_{n} \cdot\left(\theta_{n}-\theta_{\pi(n)}\right)=\beta_{n} F_{n}, \quad \forall n \in \mathcal{N}_{t}, t \geq 1 \\
& 0 \leq Z_{n} \cdot \theta_{n} \quad \forall n \in \mathcal{N}_{T} .
\end{array}
$$

The writer's problem deals with the actual cost of a selffinanced portfolio process that replicates the pay-outs that will be due to the holder of the claim without risking negative terminal wealth positions in any of the states of the market. Among such portfolios, one giving the smallest cost portfolio is an optimal portfolio, and its cost is the minimum amount the writer should charge to sell the claim. The buyer's problem is simply the opposite. He/she is prepared to pay at most the current value of the optimal portfolio that replicates the proceeds from the claim in a self-financed manner and at no risk of negative terminal positions. We refer to the optimal values of the above problems commonly as "no-arbitrage bounds".

Under the assumption of no-arbitrage in the market, these problems lead to the following pricing interval obtained using linear programming duality - see King (2002) for details:

$$
\left[\frac{1}{\beta_{0}} \min _{q \in \mathcal{Q}} \mathbb{E}^{Q}\left(\sum_{t=1}^{T} \beta_{t} F_{t}\right) ; \frac{1}{\beta_{0}} \max _{q \in \mathcal{Q}} \mathbb{E}^{Q}\left(\sum_{t=1}^{T} \beta_{t} F_{t}\right)\right],
$$

where $\mathcal{Q}$ represents the set of all measures that make the price process a martingale (not necessarily equivalent to $P$ ). More precisely, $\mathcal{Q}$ is the set of $q_{n}, n \in \mathcal{N}$ described as

$$
\begin{aligned}
\mathcal{Q} & =\left\{q_{n} \mid q_{n} \geq 0, n \in \mathcal{N}_{T}, q_{n} Z_{n}\right. \\
& \left.=\sum_{m \in \mathcal{S}(n)} q_{m} Z_{m}, n \in \mathcal{N}_{t}, t \leq T-1, q_{0}=1\right\} .
\end{aligned}
$$

\section{Pricing bounds using the Arbitrage-adjusted sharpe ratio}

The main question of the present section is: can we find sharper pricing bounds to contingent claims than the noarbitrage bounds obtained by solving problems WA and BA? We may begin by relaxing the requirement that final wealth positions $Z_{n} \cdot \theta_{n}$ be non-negative for all $n \in \mathcal{N}_{T}$. To this end, we propose to split every final wealth position $Z_{n} \cdot \theta_{n}$ into the sum of a non-negative component $v_{n}$ and an unrestricted-insign (free) component $x_{n}$ :

$Z_{n} \cdot \theta_{n}=v_{n}+x_{n} \quad \forall n \in \mathcal{N}_{T}$,

and to replace the inequalities $Z_{n} \cdot \theta_{n} \geq 0, \forall n \in \mathcal{N}_{T}$ in problems WA and BA by the Eq. (2). This relaxation of the pricing problem might result in extremely large negative final wealth positions that are unacceptable for the person who is trying to construct a sequence of hedging portfolios for a given contingent claim. To limit the magnitude of negative final wealth positions, we impose the restriction that the expected value of free component $x_{n}$ of the final wealth remain nonnegative and be at least a positive multiple of its standard deviation. In other words, if $x_{n}$, for $n \in \mathcal{N}_{T}$ represent the realizations of random variable $X$, using some positive real $\lambda$ we impose the restriction

$\mathbb{E}(X) \geq \lambda \sqrt{V(X)}$,

where $\mathbb{E}(X)$ denotes expectation and $V(X)$ denotes variance of $X$. More precisely, in our setting this restriction translates into

$\sum_{n \in \mathcal{N}_{T}} p_{n} x_{n} \geq \lambda \sqrt{\sum_{n \in \mathcal{N}_{T}} p_{n}\left(x_{n}-\sum_{n \in \mathcal{N}_{T}} p_{n} x_{n}\right)^{2}}$.

We note that inequality (3) could be also be viewed as $\frac{E(X)}{\sqrt{V(X)}} \geq$ $\lambda$ which is precisely a lower-bound restriction on the Sharpe ratio (Černý, 2003; Judice, 2005) of the unrestricted-in-sign component of the final wealth position. We term the Sharpe ratio of the unrestricted-in-sign component of the final wealth position the "arbitrage-adjusted Sharpe ratio". Put in other words, we take the viewpoint of the investor who is willing to give up the requirement of non-negative final wealth in all states of nature, and can accept some negative wealth positions provided that they satisfy restriction (4). The term "arbitrageadjusted" reflects the feature that the new pricing problems introduced below tend to give identical results with no-arbitrage pricing problems WA and BA in the limit when $\lambda$ approaches infinity as we explain in Remark 1 following Theorem 2 . This development is motivated by the classical problem of finance where investors are interested in identifying zero cost investment opportunities with the highest possible Sharpe ratio. In our context, a writer who identifies a sequence of portfolio holdings resulting in non-negative wealth positions in all states of nature while hedging the cash outlays of a contingent claim can be thought of having achieved an infinite Sharpe ratio as follows. The writer can set the $X$ component of the final wealth position to zero in all states of nature while keeping the nonnegative wealth positions in the variables $v_{n}$. This results in both zero expected value and zero variance of $X$, which may be combined with an infinitely large $\lambda$. Hence, we can identify a sequence of hedging portfolios for a contingent claim resulting in non-negative final wealth positions with an arbitrarily large $\lambda$ in (3), and hence with an infinite arbitrage-adjusted Sharpe ratio.

The writer of a contingent claim is now concerned with the following question: what is the actual cost of a self-financed portfolio process that replicates the pay-outs that will be due to the holder of the claim while risking some negative terminal wealth positions held in check by a restriction on the Sharpe ratio? Among such portfolios, one giving the smallest cost portfolio is an optimal portfolio, and its cost is the minimum amount the writer should charge to sell the claim. The buyer's 
problem is simply the opposite. What is the maximum he/she should be prepared to pay to acquire a particular claim? At most it is the current value of the optimal portfolio that replicates the proceeds from the claim in a self-financed manner and allowing some negative terminal positions controlled by a restriction on Sharpe ratio.

The writer's problem for financing a contingent claim $F$ (referred to as WSP) is

$$
\begin{array}{ll}
\min & Z_{0} \cdot \theta_{0} \\
\text { s.t. } & Z_{n} \cdot\left(\theta_{n}-\theta_{\pi(n)}\right)=-\beta_{n} F_{n}, \quad \forall n \in \mathcal{N}_{t}, t \geq 1 \\
& \sum_{n \in \mathcal{N}_{T}} p_{n} x_{n}-\lambda \sqrt{\sum_{n \in \mathcal{N}_{T}} p_{n}\left(x_{n}-\sum_{n \in \mathcal{N}_{T}} p_{n} x_{n}\right)^{2} \geq 0} \\
& v_{n}+x_{n}=Z_{n} \cdot \theta_{n} \quad \forall n \in \mathcal{N}_{T}, \\
& v_{n} \geq 0 \quad \forall n \in \mathcal{N}_{T},
\end{array}
$$

while the buyer's problem of finding the maximum acceptable price (referred to as BSP) is

$$
\begin{array}{ll}
\max & -Z_{0} \cdot \theta_{0} \\
\text { s.t. } & Z_{n} \cdot\left(\theta_{n}-\theta_{\pi(n)}\right)=\beta_{n} F_{n}, \quad \forall n \in \mathcal{N}_{t}, t \geq 1 \\
& \sum_{n \in \mathcal{N}_{T}} p_{n} x_{n}-\lambda \sqrt{\sum_{n \in \mathcal{N}_{T}} p_{n}\left(x_{n}-\sum_{n \in \mathcal{N}_{T}} p_{n} x_{n}\right)^{2} \geq 0,} \\
& v_{n}+x_{n}=Z_{n} \cdot \theta_{n} \quad \forall n \in \mathcal{N}_{T}, \\
& v_{n} \geq 0 \quad \forall n \in \mathcal{N}_{T} .
\end{array}
$$

There are two crucial properties to problems WSP and BSP. The first property is that any sequence of portfolio holdings $\theta_{0}, \theta_{1}, \ldots, \theta_{n}$ that replicate the cash flows of the contingent claim (i.e. satisfying the first constraint of problem WA and BA, respectively) and resulting in non-negative terminal wealth positions for all $n \in \mathcal{N}_{T}$ yields a feasible solution for the corresponding problem WSP (or, BSP) for every choice of $\lambda$. This property follows by setting $v_{n}$ equal to $Z_{n} \cdot \theta_{n}$ for all $n \in \mathcal{N}_{T}$ (which are all non-negative), and all the $x_{n}$ 's to zero.

The second crucial property is that when $\lambda$ increases to $\infty$, one effectively solves the duals to problems WA and BA of the previous section, respectively, after suppressing the variables $x_{n}$, i.e. we reach the arbitrage pricing theory in the limit as $\lambda$ increases without bound. The easiest way to see this is through convex programming duality as we shall do in Remark 1 following Theorem 2 .

Notice that both problems above are convex optimization (in fact, conic) problems. We analyze first the writer's problem using Lagrange duality since the analysis of the buyer's problem is similar. Forming the Lagrange function and carrying out the separate maximizations over the variables $x_{n}, v_{n}, \Theta$, respectively, we obtain the following dual problem in the variables $\left\{\omega_{n}\right\}_{n \in \mathcal{N}_{T}},\left\{q_{n}\right\}_{n \in \mathcal{N}_{T}}$ :

$\max \sum_{n>0} \beta_{n} q_{n} F_{n}$

subject to

$\|\tilde{\omega}\|_{2} \leq \lambda$
$q_{n}=p_{n}\left(1+\tilde{p}^{T} \tilde{\omega}\right)-\sqrt{p_{n}} \omega_{n}, \quad \forall n \in \mathcal{N}_{T}$,

$q_{m} Z_{m}=\sum_{n \in \mathcal{S}(m)} q_{n} Z_{n}, \quad \forall m \in \mathcal{N}_{t}, 0 \leq t \leq T-1$

$q_{0}=1$,

$q_{n} \geq 0, \quad \forall n \in \mathcal{N}_{T}$,

where $\tilde{p}$ is the vector with components $\sqrt{p_{n}}$, and $\tilde{\omega}$ is the vector with components $\left\{\omega_{n}\right\}_{n \in \mathcal{N}_{T}}$. The derivation of this dual problem is given in the Appendix. The dual of the buyer's problem is simply

$\min \sum_{n>0} \beta_{n} q_{n} F_{n}$

subject to (6)-(10), i.e. minimizing the same objective over the same set of constraints. Using the property that the first entry of $Z_{n}$ is equal to 1 for all $n$, we have that the vector $q_{n}$ is a probability measure that makes the price process $\left\{Z_{t}\right\}$ a martingale. Now, observe that one can solve the Eq. (7) for $\omega_{n}$, $n \in \mathcal{N}_{T}$, and obtain the following solution:

$\omega_{n}=-\sqrt{p_{n}}\left(q_{n} / p_{n}-1\right), \quad n \in \mathcal{N}_{T}$.

Hence, it is immediately seen after elimination of $\omega_{n}$ 's that the constraint (6) can be re-written as

$\sqrt{\sum_{n \in \mathcal{N}_{T}} p_{n}\left(\frac{q_{n}}{p_{n}}-1\right)^{2}} \leq \lambda$.

The above constraint is a second-order (Lorentz) cone inequality in the terminology of Ben-Tal and Nemirovski (2001) for the vector with $\left|\mathcal{N}_{T}\right|+1$ components where the first $\left|\mathcal{N}_{T}\right|$ components have the form $\sqrt{p_{n}}\left(\frac{q_{n}}{p_{n}}-1\right)$, and the last component is $\lambda$.

Now, we can state the following result whose proof follows from the previous development and the Conic Duality Theorem - see Ben-Tal and Nemirovski (2001).

Theorem 2. Assuming that problems BSP and WSP possess strictly feasible solutions ${ }^{1}$ we have

1. the minimum price charged by a writer of contingent claim $F$ desiring an arbitrage-adjusted Sharpe ratio of terminal wealth positions equal to $\lambda$ or higher is given by

$$
\frac{1}{\beta_{0}} \max _{q \in \mathcal{Q}(\lambda)} \mathbb{E}^{Q}\left(\sum_{t=1}^{T} \beta_{t} F_{t}\right),
$$

where the set $\mathcal{Q}(\lambda)$ is the set of all martingale measures for the discrete price process $\left\{Z_{t}\right\}$ satisfying the side condition (12),

\footnotetext{
1 Conic (second-order cone) duality theory has some features that can lead to pathological cases of primal-dual problems. Since these cases are not essential to our development, we leave them aside, and refer the interested reader to the excellent book by Ben-Tal and Nemirovski (2001), Lecture 2 for an extensive treatment of conic duality theory. The assumption of strict feasibility helps us to avoid these cases. Using Theorem 1, this assumption also implies that the market has no arbitrage.
} 
2. the maximum price acceptable to the buyer seeking at least an arbitrage-adjusted Sharpe ratio of $\lambda$ from the terminal wealth positions is given by

$$
\frac{1}{\beta_{0}} \min _{q \in \mathcal{Q}(\lambda)} \mathbb{E}^{Q}\left(\sum_{t=1}^{T} \beta_{t} F_{t}\right) .
$$

Proof. From the preceding derivation of the dual problem, we can formulate the dual problem as simply

$$
\min \sum_{n>0} \beta_{n} q_{n} F_{n}
$$

subject to (8), (9), (10) and (12). Let $\mathcal{Q}(\lambda)$ be the set of $q_{n}$ satisfying (8), (9), (10) and (12). This gives the dual problem as expressed in part 1. The rest is a consequence of the Conic Duality Theorem; see Corollary 2.4.1 of Ben-Tal and Nemirovski (2001).

Remark 1. Notice that when $\lambda$ increases to $\infty$, one effectively solves the no-arbitrage pricing problems WA and BA of the previous section through their respective duals, since the constraint (12) disappears, and the sets $\mathcal{Q}(\lambda)$ coincides with the set $\mathcal{Q}$ of the previous section, which corresponds to dual of the no-arbitrage pricing problem.

Remark 2. The strict feasibility assumption of the previous theorem means the following. For the primal problem WSP, a strictly feasible solution is a sequence of portfolio holdings $\theta_{0}, \theta_{1}, \ldots, \theta_{|\mathcal{N}|}$ with the associated $x_{n}$ for all $n \in \mathcal{N}_{T}$ such that the constraint

$\sum_{n \in \mathcal{N}_{T}} p_{n} x_{n}-\lambda \sqrt{\sum_{n \in \mathcal{N}_{T}} p_{n}\left(x_{n}-\sum_{n \in \mathcal{N}_{T}} p_{n} x_{n}\right)^{2}} \geq 0$

holds as a strict inequality with $v_{n}>0$ for all $n \in \mathcal{N}_{T}$. For the dual problem to WSP, strict feasibility means the existence of $q_{n}>0, n \in \mathcal{N}_{T}$, satisfying (8) and (9) such that the restriction (12) holds as a strict inequality. Similar comments apply to BSP and its dual.

Remark 3. As an important consequence of the theorem, we obtain the following pricing interval whose end-points correspond to the maximum price a potential buyer is willing to pay to acquire the contingent claim and the minimum price the writer of the contingent claim is willing to charge, and all this while limiting the risk of falling short in the sense of a restriction on the arbitrage-adjusted Sharpe ratio of terminal wealth at level $\lambda$ :

$\left[\frac{1}{\beta_{0}} \min _{q \in \mathcal{Q}(\lambda)} \mathbb{E}^{Q}\left(\sum_{t=1}^{T} \beta_{t} F_{t}\right) ; \frac{1}{\beta_{0}} \max _{q \in \mathcal{Q}(\lambda)} \mathbb{E}^{Q}\left(\sum_{t=1}^{T} \beta_{t} F_{t}\right)\right]$.

The above interval is a narrower interval in width compared to the arbitrage-free pricing interval as a result of the observation that both the writer and the buyer's dual problems have feasible sets contained in the feasible set of the arbitragebound problems of the previous section.

Remark 4. As $\lambda$ is decreased, the dual feasible set shrinks since we have $\mathcal{Q}\left(\lambda_{1}\right) \subseteq \mathcal{Q}\left(\lambda_{2}\right)$ for $\lambda_{1} \leq \lambda_{2}$. Therefore, we expect to be able to decrease $\lambda$ to a limiting value beyond which the feasible set $\mathcal{Q}(\lambda)$ is empty. To compute this limiting point, one can simply solve the conic optimization problem referred to as ML (where $\lambda$ is now a variable)

$\min _{q, \lambda}\{\lambda:(8),(9),(10),(12)\}$.

Notice that the problem ML can be solved as a strictly convex quadratic programming problem after eliminating the variable $\lambda$ and squaring the objective function:

$\min _{q}\left\{\sum_{n \in \mathcal{N}_{T}} p_{n}\left(\frac{q_{n}}{p_{n}}-1\right)^{2}:(8)-(10)\right\}$.

The above problem has unique optimal solution due to strict convexity of the objective function.

\section{Proportional transaction costs}

In financial markets the investor typically has to pay transaction costs proportional to the magnitude of the trade in risky assets; see Cvitanić and Karatzas (1996), Edirisinghe, Naik, and Uppal (1993) and Soner, Shreve, and Cvitanić (1995) for discussions and related work on the subject. In this section we show how the approach of the previous section can be appropriately modified to take this aspect of financial markets into account. While it is well-known that there exists no nontrivial hedging portfolio for European options in a continuous time trading setting under proportional transaction costs as shown in Soner et al. (1995), one can obtain non-trivial hedges in discrete time with the approach of the present paper.

Let $\bar{\theta}_{n}$ represent the sub-vector with $J$ components of the vector $\theta_{n}$, obtained by excluding the first component corresponding to the riskless asset. Let $\bar{Z}_{n}$ have an identical definition. We denote the transaction cost factor by $\eta$. Now, the writer's problem for financing a contingent claim $F$ under a restriction $\lambda$ on the arbitrage-adjusted Sharpe ratio is (referred to as WSPTC)

$$
\begin{array}{ll}
\min & Z_{0} \cdot \theta_{0}+\eta \bar{Z}_{0} \cdot\left|\bar{\theta}_{0}\right| \\
\text { s.t. } & Z_{n} \cdot\left(\theta_{n}-\theta_{\pi(n)}\right)+\eta \bar{Z}_{n} \cdot\left|\bar{\theta}_{n}-\bar{\theta}_{\pi(n)}\right|=-\beta_{n} F_{n}, \\
& \forall n \in \mathcal{N}_{t}, t \geq 1 \\
& \sum_{n \in \mathcal{N}_{T}} p_{n} x_{n}-\lambda \sqrt{\sum_{n \in \mathcal{N}_{T}} p_{n}\left(x_{n}-\sum_{n \in \mathcal{N}_{T}} p_{n} x_{n}\right)^{2}} \geq 0 \\
& v_{n}+x_{n}=Z_{n} \cdot \theta_{n} \quad \forall n \in \mathcal{N}_{T}, \\
& v_{n} \geq 0 \quad \forall n \in \mathcal{N}_{T},
\end{array}
$$

while the buyer's problem of finding the maximum acceptable price is (referred to as BSPTC)

$$
\begin{array}{ll}
\max & -Z_{0} \cdot \theta_{0}-\eta \bar{Z}_{0} \cdot\left|\bar{\theta}_{0}\right| \\
\text { s.t. } \quad & Z_{n} \cdot\left(\theta_{n}-\theta_{\pi(n)}\right)+\eta \bar{Z}_{n} \cdot\left|\bar{\theta}_{n}-\bar{\theta}_{\pi(n)}\right|=\beta_{n} F_{n}, \\
& \forall n \in \mathcal{N}_{t}, t \geq 1 \\
& \sum_{n \in \mathcal{N}_{T}} p_{n} x_{n}-\lambda \sqrt{\sum_{n \in \mathcal{N}_{T}} p_{n}\left(x_{n}-\sum_{n \in \mathcal{N}_{T}} p_{n} x_{n}\right)^{2}} \geq 0
\end{array}
$$




$$
\begin{aligned}
& v_{n}+x_{n}=Z_{n} \cdot \theta_{n} \quad \forall n \in \mathcal{N}_{T}, \\
& v_{n} \geq 0 \quad \forall n \in \mathcal{N}_{T} .
\end{aligned}
$$

We transform the above problems into equivalent problems without using the absolute value operator, and by a well-known reformulation involving auxiliary variables and inequalities. Using appropriately dimensioned vectors $t_{0}, t_{1}, \ldots, t_{|\mathcal{N}|}$ this reformulation yields

$$
\begin{array}{ll}
\min & Z_{0} \cdot \theta_{0}+\eta \bar{Z}_{0} \cdot t_{0} \\
\text { s.t. } & -t_{0} \leq \bar{\theta}_{0} \leq t_{0} \\
& Z_{n} \cdot\left(\theta_{n}-\theta_{\pi(n)}\right)+\eta \bar{Z}_{n} \cdot t_{n}=-\beta_{n} F_{n}, \quad \forall n \in \mathcal{N}_{t}, t \geq 1 \\
& -t_{n} \leq \bar{\theta}_{n}-\bar{\theta}_{\pi(n)} \leq t_{n} \quad \forall n \in \mathcal{N}_{t}, t \geq 1 \\
& \sum_{n \in \mathcal{N}_{T}} p_{n} x_{n}-\lambda \sqrt{\sum_{n \in \mathcal{N}_{T}} p_{n}\left(x_{n}-\sum_{n \in \mathcal{N}_{T}} p_{n} x_{n}\right)^{2}} \geq 0 \\
& v_{n}+x_{n}=Z_{n} \cdot \theta_{n} \quad \forall n \in \mathcal{N}_{T}, \\
& v_{n} \geq 0 \quad \forall n \in \mathcal{N}_{T}, \\
& t_{n} \geq 0 \quad \forall n \in \mathcal{N}_{t}, t \geq 1
\end{array}
$$

for WSPTC. The buyer's problem BSPTC is transformed similarly.

Using Lagrange duality, we obtain the following dual problems to WSPTC and BSPTC, respectively over variables $q_{n}, n \in \mathcal{N}_{t}, 0 \leq t \leq T, \alpha_{n}, \gamma_{n} \in \mathbb{R}^{m}, n \in \mathcal{N}_{t}, 0 \leq t \leq T-1$ :

$$
\max (\min ) \sum_{n>0} \beta_{n} q_{n} F_{n}
$$

subject to

$$
\begin{aligned}
& \|\tilde{\omega}\|_{2} \leq \lambda, \\
& q_{n}=p_{n}\left(1+\tilde{p}^{T} \tilde{\omega}\right)-\sqrt{p_{n}} \omega_{n}, \quad \forall n \in \mathcal{N}_{T}, \\
& q_{m}=\sum_{n \in \mathcal{S}(m)} q_{n}, \quad \forall m \in \mathcal{N}_{t}, 0 \leq t \leq T-1 \\
& q_{m} \bar{Z}_{m}-\alpha_{m}+\gamma_{m}=\sum_{n \in \mathcal{S}(m)} q_{n} \bar{Z}_{n}, \\
& \quad \forall m \in \mathcal{N}_{t}, 0 \leq t \leq T-1 \\
& \alpha_{m}+\gamma_{m} \leq \eta \bar{Z}_{m} \quad \forall m \in \mathcal{N}_{t}, 0 \leq t \leq T-1 \\
& \alpha_{m}, \gamma_{m} \geq 0 \quad \forall m \in \mathcal{N}_{t}, 0 \leq t \leq T-1 \\
& q_{0}=1, \\
& q_{n} \geq 0, \quad \forall n \in \mathcal{N}_{T} .
\end{aligned}
$$

After elimination of the $\omega_{n}$ variables as in the previous section, we find that the dual problems consist in maximizing (or, minimizing) the function $\sum_{n>0} \beta_{n} q_{n} F_{n}$ over all the measures $q_{n}, n \in \mathcal{N}_{T}$ (and $q_{n} \geq 0, \forall n \in \mathcal{N}_{t}, 0 \leq t \leq T-1$ ) and vectors $(\alpha, \gamma)=\left(\alpha_{n}, \gamma_{n} \in \mathbb{R}_{+}^{m}, n \in \mathcal{N}_{t}, 0 \leq t \leq T-1\right)$ satisfying (12), (17), (18), (19) and (20). Let us denote the feasible set of the above problems by $\mathcal{Q}_{\eta}(\lambda)$. Then we have the following result, the proof of which is similar to the proof of Theorem 2 , therefore omitted.

Theorem 3. Assuming that problems BSPTC and WSPTC possess strictly feasible solutions we have
1. the minimum price charged by a writer of contingent claim $F$ desiring an arbitrage-adjusted Sharpe ratio of terminal wealth positions equal to $\lambda$ or higher in the presence of proportional transaction costs for trades in the risky assets is given by

$$
\frac{1}{\beta_{0}} \max _{q, \alpha, \gamma \in \mathcal{Q}_{\eta}(\lambda)} \mathbb{E}^{Q}\left(\sum_{t=1}^{T} \beta_{t} F_{t}\right),
$$

2. the maximum price acceptable to the buyer seeking at least an arbitrage-adjusted Sharpe ratio of $\lambda$ from the terminal wealth positions in presence of proportional transaction costs for trades in the risky assets is given by

$$
\frac{1}{\beta_{0}} \min _{q, \alpha, \gamma \in \mathcal{Q}_{\eta}(\lambda)} \mathbb{E}^{Q}\left(\sum_{t=1}^{T} \beta_{t} F_{t}\right) .
$$

Remark 5. For the primal problem WSPTC, a strictly feasible solution is a sequence of portfolio holdings $\theta_{0}, \theta_{1}, \ldots, \theta_{|\mathcal{N}|}$ with the associated $x_{n}$ for all $n \in \mathcal{N}_{T}$ such that the constraints

$$
\begin{aligned}
& \sum_{n \in \mathcal{N}_{T}} p_{n} x_{n}-\lambda \sqrt{\sum_{n \in \mathcal{N}_{T}} p_{n}\left(x_{n}-\sum_{n \in \mathcal{N}_{T}} p_{n} x_{n}\right)^{2}} \geq 0 \\
& -t_{0} \leq \bar{\theta}_{0} \leq t_{0} \\
& -t_{n} \leq \bar{\theta}_{n}-\bar{\theta}_{\pi(n)} \leq t_{n} \quad \forall n \in \mathcal{N}_{t}, t \geq 1
\end{aligned}
$$

hold as strict inequalities with $v_{n}>0$ for all $n \in \mathcal{N}_{T}$, and $t_{n}>0$ for all $n \in \mathcal{N}_{t}, t \geq 1$. For the dual problem to WSPTC, strict feasibility means the existence of $q_{n}>0$, $n \in \mathcal{N}_{T}$, satisfying (17)-(18) such that the restrictions (12), (19), (20) hold as strict inequalities. Similar comments are valid for BSPTC and its dual.

Remark 6. Notice that $\mathcal{Q}(\lambda) \subseteq \mathcal{Q}_{\eta}(\lambda)$. Hence, we expect the price bounds to widen in the presence of transactions costs.

Remark 7. To find the limiting value of $\lambda$ we solve the problem MLTR below as a strictly convex quadratic programming problem as in the previous section:

$\min _{q, \alpha, \gamma}\left\{\sum_{n \in \mathcal{N}_{T}} p_{n}\left(\frac{q_{n}}{p_{n}}-1\right)^{2}:(17)-(22)\right\}$.

Our results show that the original probability measure $P$ plays a role in our asset pricing procedures, whereas in arbitragebased theory the measure $P$ does not appear except for the fact that the martingale measure used in valuation is drawn from the closure of the set of martingale measures equivalent to the original measure (King, 2002). This dependence can be alleviated to some extent by allowing the specification of several original measures in the formulation of pricing problems. This approach, equivalent to a worst-case approach with discrete scenarios, (see e.g., Chapter 6 of Rüstem and Howe (2002)) is not presented here for lack of space. 


\section{Computational results with $S \& P 500$ options}

In this section we demonstrate how the models advocated in the previous sections can be used to determine the pricing interval for an option. For simplicity of exposition we use the basic pricing models of Section 3 with a slight modification involving the "calibrated option bounds" model proposed by King et al. (2005) for comparison with the no-arbitrage pricing methods.

\subsection{Calibrated option bounds}

In the setting of King et al. (2005) liquid options traded in the market are used as benchmarks for hedging purposes in addition to securities. These liquid options give the investor the possibility of forming buy-and-hold strategies in the hedging portfolio sequence. In other words, every liquid option can be bought or shorted by the investor at time zero with the purpose of hedging a contingent claim, and no intermediary trading is available for these options. Assuming there are $K$ such liquid options, we denote them by $G^{k}, k=1, \ldots, K$. Bid and ask prices observed in the market at time 0 for option $k$ are denoted by $C_{b}^{k}$ and $C_{a}^{k}$, respectively, with the latter greater than or equal to the former. We use $G_{n}^{k}$ to denote the payoff of option $k$ at node $n$ of the scenario three, and $G_{n}$ the vector of option payoffs at node $n$. The non-negative $K$-vectors $\xi_{+}$and $\xi$ - denote, respectively, the long and short initial buy-and-hold positions in the liquid options. We assume a zero interest rate, therefore we have $S_{n}^{0}=1, \forall n \in \mathcal{N}$. Now, the writer's problem WSP should be modified as

$$
\begin{array}{ll}
\min & S_{0} \cdot \theta_{0}+C_{a} \cdot \xi_{+}-C_{b} \cdot \xi_{-} \\
\text {s.t. } & S_{n} \cdot\left(\theta_{n}-\theta_{\pi(n)}\right)=G_{n} \cdot\left(\xi_{+}-\xi_{-}\right)-F_{n}, \\
& \forall n \in \mathcal{N}_{t}, t \geq 1 \\
& \sum_{n \in \mathcal{N}_{T}} p_{n} x_{n}-\lambda \sqrt{\sum_{n \in \mathcal{N}_{T}} p_{n}\left(x_{n}-\sum_{n \in \mathcal{N}_{T}} p_{n} x_{n}\right)^{2} \geq 0} \\
& v_{n}+x_{n}=S_{n} \cdot \theta_{n}, \quad \forall n \in \mathcal{N}_{T}, \\
& v_{n} \geq 0, \quad \forall n \in \mathcal{N}_{T} \\
& \xi_{+}, \xi_{-} \geq 0 .
\end{array}
$$

By following the steps of Section 3 it is easy to verify that the dual problem of the previous program is

$$
\begin{array}{ll}
\max & \sum_{n>0} q_{n} F_{n} \\
\text { s.t. } & \sum_{n \in \mathcal{N}_{T}} p_{n}\left(\frac{q_{n}}{p_{n}}-1\right)^{2} \leq \lambda \\
& q_{m} S_{m}=\sum_{n \in \mathcal{S}(m)} q_{n} S_{n}, \quad \forall m \in \mathcal{N}_{t}, 0 \leq t \leq T-1 \\
& q_{0}=1 \\
& \sum_{n \in \mathcal{N} \backslash \mathcal{N}_{0}} q_{n} G_{n} \leq C_{a} \\
& \sum_{n \in \mathcal{N} \backslash \mathcal{N}_{0}} q_{n} G_{n} \geq C_{b} \\
& q_{n} \geq 0, \quad \forall n \in \mathcal{N}_{T} .
\end{array}
$$

The modifications required for the buyer's problem are similar. All the results of Section 3 can be re-iterated for the above primal and dual problems, after the necessary modifications.

\subsection{Numerical results}

We adopt the experimental setting of King et al. (2005) in our numerical experiments. We use 48 European options written on the $S \& P 500$ index, shown in Table 1 in our computational experimentation. The option data were available in the market on September 10, 2002. The first 21 of the options are call options, and the remaining are put options. Strikes and maturities as well as actual bid and ask prices (columns $C_{b}$ and $C_{a}$ ) of these options are given in Table 1. We compute "calibrated" pricing bounds for each option. This means that the buyer or writer of the option can include buyand-hold positions in the 47 remaining options into his/her hedge portfolio sequence.

We use two different settings: one with four trading periods and the other with five periods. In the four periods setting, we assume that investors can trade at days 0, 17, 37 and 100 (the maturity dates of the options), while in the five periods setting, we assume trading days are $0,8,17,37$ and 100. Since going to five periods does not change the results much, we report our full results for the four-period model, which already provides substantial improvement over the no-arbitrage pricing models in some cases, while we give a few illustrative results with five periods in the interest of keeping the length of the paper reasonable.

We use $S=\left(1, S^{1}\right)$ as the traded securities. We generate a scenario tree by the Gauss-Hermite process as described in King et al. (2005), Omberg (1988) and Pennanen and Koivu (2002). We assume that the value $S^{1}$ of the $S \& P 500$ index evolves as a geometric Brownian motion with daily drift $d$ and volatility $\sigma$. Let $l$ be the length of period $t$ in days. Then, the $\operatorname{logarithm} \zeta_{t}=\ln S_{t}^{1}$ evolves according to

$\zeta_{t}=\zeta_{t-1}+d_{t}+\epsilon_{t}$,

where $d_{t}=l_{t} d$, and $\epsilon_{t}$ is normally distributed with zero mean and standard deviation $\sigma_{t}=\sqrt{l_{t}} \sigma$. Using given parameters $\zeta_{0}$, the initial value of $\zeta, l_{t}, t=1, \ldots, T, d$ and $\sigma$, we construct a scenario tree approximation to the stochastic process $\zeta_{t}$ using Gauss-Hermite quadrature to obtain a sample $\left(\epsilon_{1}^{i_{1}}\right)_{i_{1}=1}^{\nu_{1}}$ of dimension $v_{1}$ with associated positive probabilities $\left(\pi_{1}^{i_{1}}\right)_{i=1}^{\nu_{1}}$. Hence, we obtain an approximation of possible values of the index at time $t=1 \mathrm{using}$ the equation $\zeta_{1}^{i_{1}}=\zeta_{0}+d_{1}+\epsilon_{1}^{i_{1}}$, $i_{1}=1, \ldots, v_{1}$. For time period $t=2$ we generate a sample $\left(\epsilon_{2}^{i_{2}}\right)_{i_{2}=1}^{\nu_{2}}$ of dimension $\nu_{2}$ with associated positive probabilities $\left(\pi_{2}^{i_{2}}\right)_{i_{2}=1}^{\nu_{2}}$ to get the possible values of the logarithmic index as $\zeta_{2}^{i_{1}, i_{2}}=\zeta_{1}^{i_{1}}+d_{2}+\epsilon_{2}^{i_{2}}, i_{1}=1, \ldots, v_{1}, i_{2}=1, \ldots, v_{2}$. Repeating this procedure for all time points up to time $T$, we obtain a scenario tree where the nodes $\mathcal{N}_{t}$ at time $t$ are labeled by the $t$-tuple $\left(i_{1}, \ldots, i_{t}\right)$. In the notation of Section 3 , we have that the set $\mathcal{N}$ of all nodes in the tree are given as the union of all nodes for each time point $t$, i.e. $\mathcal{N}=$ 
Table 1

Computational results on $S \& P 500$ options $(\lambda=5.7)$

\begin{tabular}{|c|c|c|c|c|c|c|c|}
\hline Option number & Type & Strike & Maturity & $C_{b}$ & $C_{a}$ & Arbitrage interval & Sharpe interval \\
\hline 1 & Call & 890 & 17 & 31.5 & 33.5 & $30.98-32.00$ & $31.27-31.76$ \\
\hline 2 & Call & 900 & 17 & 24.4 & 26.4 & $24.67-26.05$ & $24.67-25.32$ \\
\hline 3 & Call & 905 & 17 & 21.2 & 23.2 & $21.79-23.08$ & $21.79-22.65$ \\
\hline 4 & Call & 910 & 17 & 18.5 & 20.1 & $18.93-20.30$ & $18.93-20.03$ \\
\hline 5 & Call & 915 & 17 & 15.8 & 17.4 & $16.08-17.51$ & $16.08-17.47$ \\
\hline 6 & Call & 925 & 17 & 11.2 & 12.6 & $10.43-12.77$ & $10.58-12.77$ \\
\hline 7 & Call & 935 & 17 & 7.6 & 8.6 & 7.68-9.09 & 7.68-9.09 \\
\hline 8 & Call & 950 & 17 & 3.8 & 4.6 & $3.39-4.79$ & $3.39-4.76$ \\
\hline 9 & Call & 955 & 17 & 3 & 3.7 & $2.99-3.89$ & $2.99-3.89$ \\
\hline 10 & Call & 975 & 17 & 0.95 & 1.45 & $0.65-1.66$ & $0.65-1.66$ \\
\hline 11 & Call & 980 & 17 & 0.65 & 1.15 & $0.66-1.44$ & $0.66-1.43$ \\
\hline 12 & Call & 900 & 37 & 42.3 & 44.3 & $40.58-42.58$ & $40.58-42.58$ \\
\hline 13 & Call & 925 & 37 & 28.2 & 29.6 & $26.38-28.38$ & $26.87-28.38$ \\
\hline 14 & Call & 950 & 37 & 17.5 & 19 & $13.82-18.98$ & $14.32-18.77$ \\
\hline 15 & Call & 875 & 100 & 77.1 & 79.1 & $75.48-77.48$ & $75.48-77.48$ \\
\hline 16 & Call & 900 & 100 & 61.6 & 63.6 & $59.88-61.88$ & $59.88-61.88$ \\
\hline 17 & Call & 950 & 100 & 35.8 & 37.8 & $32.27-39.29$ & $33.81-38.90$ \\
\hline 18 & Call & 975 & 100 & 26 & 28 & $23.71-28.74$ & $24.99-28.74$ \\
\hline 19 & Call & 995 & 100 & 19.9 & 21.5 & $17.73-22.48$ & $18.90-22.48$ \\
\hline 20 & Call & 1025 & 100 & 12.6 & 14.2 & 8.02-16.44 & $10.07-15.99$ \\
\hline 21 & Call & 1100 & 100 & 3.4 & 3.8 & $0.00-12.80$ & $3.91-9.85$ \\
\hline 22 & Put & 750 & 17 & 0.4 & 0.6 & $0.00-1.15$ & $0.55-1.13$ \\
\hline 23 & Put & 790 & 17 & 1 & 1.3 & $0.87-1.44$ & $1.01-1.19$ \\
\hline 24 & Put & 800 & 17 & 1.3 & 1.65 & $1.16-1.74$ & $1.21-1.48$ \\
\hline 25 & Put & 825 & 17 & 2.5 & 2.85 & $2.18-2.86$ & $2.33-2.75$ \\
\hline 26 & Put & 830 & 17 & 2.6 & 3.1 & $2.74-3.15$ & $2.78-3.08$ \\
\hline 27 & Put & 840 & 17 & 3.4 & 3.8 & $3.20-3.90$ & $3.44-3.80$ \\
\hline 28 & Put & 850 & 17 & 3.9 & 4.7 & $4.40-4.80$ & $4.41-4.76$ \\
\hline 29 & Put & 860 & 17 & 5.5 & 5.8 & $4.61-5.94$ & $5.41-5.79$ \\
\hline 30 & Put & 875 & 17 & 7.2 & 7.8 & $6.80-7.98$ & $7.66-7.98$ \\
\hline 31 & Put & 885 & 17 & 9.4 & 10.4 & $9.97-10.78$ & $10.25-10.73$ \\
\hline 32 & Put & 750 & 37 & 5.5 & 5.9 & $3.80-6.64$ & $3.80-5.53$ \\
\hline 33 & Put & 775 & 37 & 6.9 & 7.7 & $6.33-7.95$ & $7.60-7.74$ \\
\hline 34 & Put & 800 & 37 & 9.3 & 10 & $7.90-11.23$ & $9.90-10.90$ \\
\hline 35 & Put & 850 & 37 & 16.7 & 18.3 & $13.49-19.53$ & $15.84-18.87$ \\
\hline 36 & Put & 875 & 37 & 23 & 24.3 & $21.77-25.65$ & $22.43-25.49$ \\
\hline 37 & Put & 900 & 37 & 31 & 33 & $32.72-34.05$ & $32.72-33.87$ \\
\hline 38 & Put & 925 & 37 & 41.8 & 43.8 & $43.62-45.02$ & $43.62-45.02$ \\
\hline 39 & Put & 975 & 37 & 73 & 75 & $72.24-76.91$ & $72.70-76.79$ \\
\hline 40 & Put & 995 & 37 & 88.9 & 90.9 & 87.09-94.08 & $87.73-92.95$ \\
\hline 41 & Put & 650 & 100 & 5.7 & 6.7 & $2.60-8.58$ & $2.72-5.75$ \\
\hline 42 & Put & 700 & 100 & 9.2 & 10.2 & $6.65-11.25$ & $10.02-10.60$ \\
\hline 43 & Put & 750 & 100 & 14.7 & 15.8 & $11.80-16.20$ & $15.02-16.05$ \\
\hline 44 & Put & 775 & 100 & 17.6 & 19.2 & $16.95-19.75$ & $17.84-19.72$ \\
\hline 45 & Put & 800 & 100 & 21.7 & 23.7 & $20.07-24.57$ & $21.04-24.45$ \\
\hline 46 & Put & 850 & 100 & 33.3 & 35.3 & $32.74-36.50$ & $32.97-36.37$ \\
\hline 47 & Put & 875 & 100 & 40.9 & 42.9 & $42.52-43.80$ & $42.52-43.78$ \\
\hline 48 & Put & 900 & 100 & 50.3 & 52.3 & $52.02-54.02$ & $52.02-54.02$ \\
\hline
\end{tabular}

$\mathcal{N}_{1} \cup \ldots \cup \mathcal{N}_{T}$. The parent node $a\left(i_{1}, \ldots, i_{t}\right)$ of $\left(i_{1}, \ldots, i_{t}\right)$ is the node labeled $\left(i_{1}, \ldots, i_{t-1}\right)$; the child nodes $\mathcal{D}\left(i_{1}, \ldots, i_{t}\right)$ of the node $\left(i_{1}, \ldots, i_{t}\right)$ is the set $\left\{\left(i_{1}, \ldots, i_{t+1}\right) \in \mathcal{N}_{t+1} \mid i_{t+1} \in\right.$ $\left.\left\{1, \ldots, v_{t+1}\right\}\right\}$. Finally the probability distribution $P$ for the leaf nodes is specified as $p\left(i_{1}, \ldots, i_{T}\right)=\pi_{1}^{i_{1}} \cdots \pi_{T}^{i_{T}}$, and $S_{n}=\mathrm{e}^{\zeta_{n}}$ for all $n \in \mathcal{N}$. As the number of branches $v$ increases, the tree converges weakly to a discrete time geometric Brownian motion as shown in Pennanen and Koivu (2002).

For four periods we assume a branching structure of $v_{1}=$ $50, v_{2}=10, v_{3}=10$ yielding 5000 leaf nodes in the tree. The resulting optimization models have approximately 10,600 equations and 11,200 variables. We use $d=0.0001, \sigma=$ 0.013175735 and $S_{0}^{1}=909.58$, which was the closing value of the index on 10.9.2002. For the five-period model we use $v_{1}=20, v_{2}=10, v_{3}=10, v_{4}=10$, resulting in 20, 000 leaf nodes and in conic optimization problems with approximately 24, 500 equations and 42, 200 variables.

We used the state-of-the-art conic interior point optimizer MOSEK (MOSEK Solver Manual, 2007) through GAMS (Brooke, Kendrick, \& Meeraus, 1992) to determine buyer's and writer's prices for each option. We exhibit the results for models in Table 1. In the table the column with the 
label "Arbitrage Interval" shows the no-arbitrage pricing intervals of King et al., which we re-obtained using our own implementation. In the experiments of King et al. using the no-arbitrage assumption, for most of the options actual prices fall in the pricing interval determined by buyer's and writer's problems. Our results show that, if the market makers use the arbitrage-adjusted Sharpe ratio restriction instead of the nonnegativity restriction on final wealth positions they can obtain tighter bounds closer to the actual prices, especially for the cases where arbitrage bounds fail to get close to actual prices (e.g., Table 1 options numbered 14, 17, 19, 20, 21, 34, $35,36,40,42,43,44,45)$. It is possible to improve further some of the results by choosing different $\lambda$ values for different problems. However, for consistency, we report results with the same value of $\lambda$ equal to 5.7. Passing to five periods, we obtain consistently good results with $\lambda=7.3$ after some preliminary experimentation. For example, with option number 3 , the no-arbitrage pricing interval is [21.06, 23.08] whereas the pricing interval from the arbitrage adjusted Sharpe ratio is $[21.76,22.98]$. The respective intervals are [15.20, 17.60], and $[15.78,17.59]$ for option number 5 , and [86.49, 94.08] and $[87.45,92.87]$ for option number 40 . For option number 42 , the no-arbitrage pricing interval is $[6.65,11.25]$ whereas the proposed interval is $[10.02,10.57]$. The improvement pattern of arbitrage-adjusted Sharpe ratio bounds over no-arbitrage bounds in narrowing the interval and getting closer to the observed market prices in a significant number of cases persists here as in the four periods case. All the optimization problems are usually solved within minutes of computing time.

\section{Discussion and future research}

While we were able to solve non-trivial conic programming instances by off-the-shelf interior point solvers, a current limitation of the approach of the present paper is the ability to handle instances corresponding to a larger number of branches and time periods - allowing a sixth trading period with ten branches from each leaf node would yield approximately half a million variables in the primal problem. This exponential size growth is typical in stochastic programming; see e.g. Sodhi (2005) and van Delft and Vial (2004). While the results we obtain with four and five trading period problems are satisfactory in the present application since the bounds obtained are close to the market prices of the options and the results show little change when passing from four periods to five periods, it may be necessary to increase the number of periods, and thus the size of the tree in other applications. ${ }^{2}$

\footnotetext{
2 To solve problems with a larger number of periods, e.g., ten periods, in an option hedging exercise with a stock and a bond, e.g. as in Edirisinghe et al (1993), one can use, for instance, three branches from each node (trinomial tree) with the current conic programming solvers. However, in the current calibrated bounds application, opening only three branches results in the impossibility of the exercise for some options, and leads to arbitrage opportunities, and thus to unbounded primal and infeasible dual problems by the violation of strict feasibility assumptions. This complication is not encountered with larger numbers of branches.
}

State and time aggregation procedures were proposed in the literature as a remedy to exponential size growth (Klaassen, 1998; Sodhi, 2005). However, these procedures usually produce upper and lower bounds to the optimal value of the original problem. Since our aim is to improve the potentially loose no-arbitrage bounds, these aggregation approaches may result in inaccuracies which may defeat the purpose of passing to Sharpe ratio based bounds in the first place. It appears more beneficial to look for algorithms that exploit the special structure of the original problem. A careful look into problems WSP and BSP reveals that these problems have a natural network structure since they are defined on a tree structure. The complicating factor is the conic constraint. Were it not for the conic constraint, one could use a network simplex algorithm to solve larger instances. A potentially promising avenue for further research can be to exploit the presence of a single conic constraint as in Erdogan and Iyengar (2006). These authors develop an algorithm based on duality for linear problems with equality and inequality constraints and a single second-order cone constraint, which corresponds to our problems WSP and BSP. However, their implementation does not exploit sparsity, and is currently limited to instances that can be considered small. An important competing approach to solving optimization problems defined on scenario trees is Dynamic Programming (DP). DP is a very powerful technique that models the overall dynamic decision process as a sequence of simpler and smaller optimization problems. In the present scenario tree setting the coupling constraints (4) in the primal problem and constraint (12) in the dual problem constitute a first impediment to the immediate application of DP techniques since these constraints involve decision variables for the entire collection of leaf nodes of the tree. Besides, DP also suffers from the exponential growth of the event tree, and explosion of state space, known as the "curse of dimensionality". A good discussion of the limitations of DP in general, and recent approximation ideas can be found in Powell (2007). It seems that successful DP applications in discrete-time financial hedging such as Bensaid, Lesne, Pagès, and Scheinkman (1992) and Edirisinghe et al. (1993) usually limit the number of branches in the tree to two or three.

In summary, we are currently not aware of any software system capable of handling larger instances than the ones we solved with the interior point algorithm of MOSEK. However, given that solving the conic programs of this paper by off-theshelf solvers was unthinkable a decade ago, further research on faster and structure exploiting algorithms is expected to increase our ability to tackle larger instances. This is an important future research question.

\section{Acknowledgments}

I am indebted to my Ph.D. student Ahmet Camc1 for his help with the computational experiments and for carefully proofreading the paper. The comments of three anonymous reviewers were also helpful in improving the presentation. 


\section{Appendix. Derivation of dual problem for WSP}

The writer's problem for financing a contingent claim $F$ that we referred to as WSP is rewritten as follows for convenience:

$$
\begin{array}{ll}
\min & Z_{0} \cdot \theta_{0} \\
\text { s.t. } & Z_{n} \cdot\left(\theta_{n}-\theta_{\pi(n)}\right)=-\beta_{n} F_{n}, \quad \forall n \in \mathcal{N}_{t}, t \geq 1 \\
& \sum_{n \in \mathcal{N}_{T}} p_{n} x_{n}=t \\
& t-\lambda \sqrt{\sum_{n \in \mathcal{N}_{T}} p_{n}\left(x_{n}-t\right)^{2}} \geq 0 \\
& v_{n}+x_{n}=Z_{n} \cdot \theta_{n} \quad \forall n \in \mathcal{N}_{T}, \\
& v_{n} \geq 0 \quad \forall n \in \mathcal{N}_{T} .
\end{array}
$$

We can view the expression $\lambda \sqrt{\sum_{n \in \mathcal{N}_{T}} p_{n}\left(x_{n}-t\right)^{2}}$ as $\lambda$ times the two-norm of the vector with $\left|\mathcal{N}_{T}\right|$ components given by $\sqrt{p_{n}}\left(x_{n}-t\right)$. Furthermore, using the dual representation of the two-norm (see pp. 275-276 of Horn and Johnson (1985), Section 5.4: Analytic properties of vector norms) which is expressed as $\lambda\|z\|_{2}=\max _{\zeta:\|\zeta\|_{2} \leq \lambda} z^{T} \zeta$ - note that one also has $-\lambda\|z\|_{2}=\min _{\zeta:\|\zeta\|_{2} \leq \lambda} z^{T} \zeta-$ we can rewrite $\lambda \sqrt{\sum_{n \in \mathcal{N}_{T}} p_{n}\left(x_{n}-t\right)^{2}}$ as $\max _{\|\omega\|_{2} \leq \lambda} \sum_{n \in \mathcal{N}_{T}} \omega_{n} \sqrt{p_{n}}\left(x_{n}-t\right)$ where we use $\omega$ to represent the vector with components $\omega_{n}$, $n \in \mathcal{N}_{T}$.

Now, the above problem has the following Lagrange dual problem:

$\max _{\|\omega\|_{2} \leq \lambda, \xi \geq 0, q, w, V} \min _{\Theta, x, v \geq 0, t} L(\Theta, V, x, v, q, w, \xi, \omega, t)$,

where

$$
\begin{aligned}
& L(\Theta, V, x, v, q, \xi, \omega, t)=Z_{0} \cdot \theta_{0} \\
& +\sum_{t=1}^{T} \sum_{n \in \mathcal{N}_{t}} q_{n}\left(Z_{n} \cdot\left(\theta_{n}-\theta_{\pi(n)}\right)+\beta_{n} F_{n}\right) \\
& +\sum_{n \in \mathcal{N}_{T}} w_{n}\left(Z_{n} \cdot \theta_{n}-v_{n}-x_{n}\right) \\
& +\xi\left(-t+\sum_{n \in \mathcal{N}_{T}} \omega_{n} \sqrt{p_{n}}\left(x_{n}-t\right)\right) \\
& +V\left(t-\sum_{n \in \mathcal{N}_{T}} p_{n} x_{n}\right) .
\end{aligned}
$$

Minimizing with respect to $\theta_{n}$, for $n \in \mathcal{N}_{t}, 0 \leq t \leq T-1$ we obtain $q_{m} Z_{m}=\sum_{n \in \mathcal{S}(m)} q_{n} Z_{n}, \forall m \in \mathcal{N}_{t}, 0 \leq t \leq$ $T-1$ which are Eq. (8) where we set $q_{0}=1$ (in fact, we artificially introduce a $q_{0}=1$ to avoid writing the above equations separately for $t=0$ and the remaining time points $t=1, \ldots, T-1)$. Minimization with respect to $\theta_{n}$, for $n \in \mathcal{N}_{T}$ yields $q_{n}=-w_{n}$, for $n \in \mathcal{N}_{T}$. Minimization with respect to $v_{n} \geq 0$ gives $w_{n} \leq 0$, for $n \in \mathcal{N}_{T}$. Therefore, we have $q_{n} \geq 0$ for all $n \in \mathcal{N}_{T}$. Minimization with respect to $x_{n}$ yields $-w_{n}+\xi \omega_{n} \sqrt{p_{n}}-V p_{n}=0$ for all $n \in \mathcal{N}_{T}$. Replacing $-w_{n}$ by $q_{n}$ we obtain $q_{n}+\xi \omega_{n} \sqrt{p_{n}}-V p_{n}=0$ for all $n \in \mathcal{N}_{T}$. Finally, minimizing over $t$ we obtain $V=\xi\left(\tilde{p}^{T} \tilde{\omega}+1\right)$, where $\tilde{\omega}$ denotes the vector with components $\omega_{n}, n \in \mathcal{N}_{T}$. Substituting this expression for $V$ in the equations $q_{n}+\xi \omega_{n} \sqrt{p_{n}}-V p_{n}=0$ for all $n \in \mathcal{N}_{T}$ we obtain, using the fact $\sum_{n \in \mathcal{N}_{T}} q_{n}=1$, that $\xi=1$. Therefore, we can replace all occurrences $\xi \omega_{n}$ by $\omega_{n}$.

\section{References}

Bensaid, B., Lesne, J., Pagès, H., \& Scheinkman, J. (1992). Derivative asset pricing with transaction costs. Mathematical Finance, 2, 63-86.

Ben-Tal, A., \& Nemirovski, A. (2001). Lectures on modern convex optimization: Analysis, algorithms and engineering applications. Philadelphia: MPS-SIAM.

Bernardo, A. E., \& Ledoit, O. (2000). Gain, loss and asset pricing. Journal of Political Economy, 108, 144-172.

Brooke, A., Kendrick, D., \& Meeraus, A. (1992). GAMS: A user's guide. San Fransisco, CA: The Scientific Press.

Carr, P., Geman, H., \& Madan, D. B. (2001). Pricing and hedging in incomplete markets. Journal of Financial Economics, 62, 131-167.

Černý, A. (2003). Generalized sharpe ratios and pricing in incomplete markets. European Finance Review, 7, 191-233.

Černý, A. (2004). Mathematical techniques in finance. Princeton, NJ: Princeton University Press.

Černý, A., \& Hodges, S. D. (2000). The theory of good-deal pricing in financial markets. In Mathematical Finance - Bachelier Congress 2000. Berlin: Springer-Verlag.

Cochrane, J. H., \& Saa-Requejo, J. (2000). Beyond arbitrage: Good-deal asset price bounds in incomplete markets. Journal of Political Economy, 108, 79-119.

Cvitanić, J., \& Karatzas, I. (1996). Hedging and portfolio optimization under transaction costs: A martingale approach. Mathematical Finance, 6(2), 133-165.

Edirisinghe, C., Naik, V., \& Uppal, R. (1993). Optimal replication of options with transaction costs and trading restrictions. Journal of Financial and Quantitative Analysis, 28, 117-138.

Erdogan, E., \& Iyengar, G. (2006). An active set method for singlecone second-order cone programs. SIAM Journal on Optimization, 17(2), $459-484$.

Harrison, J. M., \& Kreps, D. M. (1979). Martingales and arbitrage in multiperiod securities markets. Journal of Economic Theory, 20, 381-408.

Horn, R. A., \& Johnson, C. R. (1985). Matrix analysis. Cambridge: Cambridge University Press.

Judice, P. (2005). Foundations and applications of good-deal pricing I: Singleperiod market models. Working paper.

King, A. J. (2002). Duality and martingales: A stochastic programming perspective on contingent claims. Mathematical Programming Series B, 91, 543-562.

King, A. J., Koivu, M., \& Pennanen, T. (2005). Calibrated Option Bounds. International Journal of Theoretical and Applied Finance, 8(2), 141-159.

MOSEK Solver Manual. (2007). Mosek ApS c/o Symbion Science Park, Fruebjergvej 3, Box 15, 2100 Copenhagen, Denmark.

Klaassen, P. (1998). Financial asset-pricing theory and stochastic programming models for asset/liability management: A synthesis. Management Science, 44(1), 31-48.

Omberg, E. (1988). Efficient discrete time jump process models in option pricing. Journal of Financial and Quantitative Analysis, 23(2), 161-174.

Pennanen, T., Koivu M. (2002). Integration quadratures in discretization of stochastic programs, In Stochastic Programming E-Print Series.

Powell, W. B. (2007). Approximate dynamic programming: Solving the curses of dimensionality. New York: Wiley.

Ross, S. (1976). The arbitrage theory of capital asset pricing. Journal of Economic Theory, 13, 341-360.

Ross, S. (1978). A simple approach to the valuation of risky streams. Journal of Business, 51, 453-475.

Rüstem, B., \& Howe, M. (2002). Algorithms for worst-case design and applications to risk management. Princeton, NJ: Princeton University Press.

Sodhi, M. (2005). LP modeling for asset-liability management: A survey of choices and simplifications. Operations Research, 53(2), 181-196. 
Soner, H. M., Shreve, S., \& Cvitanić, J. (1995). There is no nontrivial hedging portfolio for option pricing with transaction costs. Annals of Applied Probability, 5, 327-355.

Staum, J. (2004). Fundamental theorems of asset pricing for good deal bounds. Mathematical Finance, 14, 141-161.

van Delft, C., \& Vial, J. P. (2004). A practical implementation of stochastic programming: An application to the evaluation of option contracts in supply chains. Automatica, 40, 743-756.

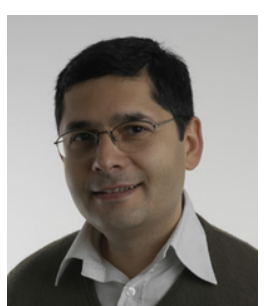

Mustafa Ç. Pınar earned M.Sc. and Ph.D. degrees in Systems Engineering from University of Pennsylvania. $\mathrm{He}$ is a professor of Industrial Engineering at Bilkent University, Ankara, Turkey. He is currently a visiting Fulbright scholar at Princeton University, USA. His research interest is optimization applied to financial valuation and planning problems. 